\title{
Pessoas em situação de rua no litoral do Piauí: um relato de experiência pelas lentes do Pet-Saúde/Interprofissionalidade
}

\author{
Homeless people in the city of Parnaíba-PI: an experience report through the Pet- \\ Saúde/Interprofissionalidade lens
}

\begin{abstract}
Personas sin hogar en la ciudad de Parnaíba-PI: un informe de experiencia a través de la lente de Pet-Saúde/Interprofissionalidade
\end{abstract}

Alexia Jade Machado Sousa ${ }^{1 *}$, Thalia Ariadne Pena Aragão', Isaac Vasconcelos Barbosa ${ }^{1}$, Joyce Maria Araújo Cavalcante ${ }^{1}$, Kerolayne de Castro Fontenele ${ }^{1}$, Shacha de Brito Véras Brejal Pereira', Beatriz de Oliveira Nobre', Geovania Vieira de Brito², Cláudio Ângelo Ventura ${ }^{1}$, Francisco Jander de Sousa Nogueira'.

\begin{abstract}
RESUMO
Objetivo: Relatar a experiência de trabalho interprofissional de acadêmicos da área de saúde junto às pessoas em situação de rua em um município do estado do Piauí. Relato de Experiência: Esse estudo trata-se de um relato de experiência do tipo descritivo qualitativo, obtido pela participação dos estudantes do PET-Saúde/Interprofissionalidade em rodas de conversa com usuários e usuárias do Centro POP em um município no litoral do Piauí. Nas falas percebemos o sofrimento dessa população: o preconceito direcionado a pessoas em situação de rua, o que é observado também em âmbito nacional. Pesquisas nacionais nos mostram que as Infecções Sexualmente Transmissíveis estão relacionadas a fatores individuais, sociais e de saúde, atreladas a condições do contexto no qual as pessoas estão inseridas, sendo alguns grupos populacionais, a exemplo das Pessoas em Situação de Rua, considerados em estado de maior vulnerabilidade. Considerações Finais: A perspectiva dos participantes que protagonizam esse estudo trata-se de um público que traz impregnado no seio de seu discurso, a denúncia de um preconceito, um desvalor e uma total desigualdade no tocante às formas de atendimento e recebimento de cuidados, bem como nas diversas formas de existência como um todo.
\end{abstract}

Palavras-chave: Pessoas em situação de rua, Atenção primária à saúde, Promoção da saúde.

\begin{abstract}
Objective: To report the experience of interprofessional work by academics in the health field with people living on the streets in a municipality in Piauí. Experience report: This study is a qualitative descriptive experience report, obtained by the participation of PET-Saúde / Interprofessional students in conversation circles with users of the POP Center in a municipality on the coast of the State of Piauí. In the speeches we perceive the suffering of this population: the prejudice established against people on the street, which is also observed at the national level. National surveys show us that Sexually Transmitted Infections are related to individual, social and health factors, linked to conditions in the context in which people are inserted, with some population groups, such as Street People, being considered vulnerable. Final Considerations: The perspective of the participants who lead this study is an audience that is impregnated within their discourse, denouncing a prejudice, a devaluation and a total inequality regarding the forms of care and receiving care, as well as in the various forms of existence as a whole.
\end{abstract}

Key words: Homeless people, Primary health care, Health promotion.

${ }_{1}$ Universidade Federal do Piauí (UFPI), Parnaíba-PI. *E-mail: alexyajade@hotmail.com

2 Secretaria Municipal de Saúde (SMS), Parnaíba-PI. 


\section{RESUMEN}

Objetivo: Informar la experiencia del trabajo interprofesional realizado por académicos en el campo de la salud con personas que viven en las calles de un municipio de Piauí. Informe de experiencia: Este estudio es un informe de experiencia descriptivo cualitativo, obtenido por la participación de estudiantes de PETSaúde / Interprofesional en círculos de conversación con usuarios del Centro POP en un municipio en la costa del Estado de Piauí. En los discursos percibimos el sufrimiento de esta población: el prejuicio establecido contra las personas en la calle, que también se observa a nivel nacional. Las encuestas nacionales nos muestran que las infecciones de transmisión sexual están relacionadas con factores individuales, sociales y de salud, vinculados a condiciones en el contexto en el que se insertan personas, y algunos grupos de población, como la gente de la calle, se consideran vulnerables. Consideraciones finales: La perspectiva de los participantes que lideran este estudio es una audiencia que está impregnada dentro de su discurso, denunciando también un prejuicio, una devaluación y una desigualdad total con respecto a las formas de atención y recibir atención. como en las diversas formas de existencia en su conjunto.

Palabras clave: Personas sin hogar, Atención primaria de salud, Promoción de la salud.

\section{INTRODUÇÃO}

A crescente globalização e o avanço tecnológico têm gerado consequências que contribuem para 0 aumento das desigualdades sociais, como também prejudicam as garantias de direitos sociais para grande parcela da população. Essa desigualdade na distribuição de bens sociais, a vulnerabilidade locacional, a insalubridade, a discriminação, o desrespeito às diferenças, são fortalecidas pelo pensamento globalizado e também pelo processo econômico vigente (COSTA APM, 2005).

Segundo Costa APM (2005) em países como o Brasil, onde não aconteceu um efetivo estabelecimento do estado de bem-estar social da população, há uma elevada tendência para que se encontrem fortes desigualdades sociais. Na realidade brasileira, as políticas sociais adotadas durante os diferentes governos tiveram uma implicação de caráter focalizado, refletindo a tendência de enfrentar os problemas sociais como fatos isolados, tendo como consequência resultados não efetivos na melhoria da qualidade de vida de parte da população, principalmente entre as pessoas em situação de rua.

Sobre esse grupo, se configura como uma "categoria social complexa e em construção, cujo lugar na cotidianidade transita entre o visível e o invisível" (PIMENTA MM, 2019), deve-se destacar que é heterogêneo, composto por pessoas com diferentes realidades, porém, com a pobreza absoluta como fator de interseção entre elas. Dentre os seus representantes, existem homens, mulheres, jovens, famílias inteiras ou grupos afetados por inúmeras e diferentes intempéries em suas histórias de vida. Além da origem econômica (principal fator), essa exclusão social existente no país também tem origem na falta de pertencimento social e possibilidades de perspectivas diferentes, além da dificuldade de acesso à informação e perda de autoestima. Essa realidade é característica do processo de exclusão social que existe no Brasil neste início de milênio (COSTA APM, 2005).

A política de Assistência Social propõe uma resolução das necessidades ligadas à situação de extrema pobreza no país, sendo inclusos a garantia de direitos, o acesso a serviços assistenciais, a reconstrução de vínculos sociais e projetos de vida para pessoas em situação de rua e sua inserção em serviços de acolhimento em repúblicas. No entanto, os principais serviços de referência socioassistenciais apresentam problemas ao lidar com essa população quando se refere a transtornos mentais e articulação com os outros serviços da rede de saúde (BORYSOW IC; FURTADO JP, 2013).

Alguns autores discorrem sobre os principais motivos que levam as pessoas a morarem nas ruas: perda de vínculos familiares, desemprego, violência, perda de algum ente querido, perda de autoestima, alcoolismo, drogadição, doença mental, dentre outros fatores. São histórias de rupturas sucessivas e que, com muita frequência, estão associadas ao uso de álcool e drogas, não só pela pessoa que está na rua, mas por outros membros da família (COSTA APM, 2005). 
Na Política Nacional para a População em Situação de Rua, instituída em 2009 - política que faz parte das Políticas de Promoção da Equidade em Saúde, sendo um conjunto de Políticas e Programas de Saúde, instituídos no âmbito do Sistema Único de Saúde - SUS; tem como princípio a equidade e contribui para promover o respeito à diversidade e garantir o atendimento integral no SUS às populações em situação de vulnerabilidade e desigualdade social. Ela também dispõe sobre essa população como um grupo heterogêneo que converge em aspectos como a pobreza extrema, os laços familiares rompidos e o fato de se utilizarem provisória ou permanentemente de locais públicos como moradia (BRASIL, 2009).

Também foram definidas diretrizes para a implantação e implementação de serviços destinados a esta população, como os Centros de Referência Especializados para População em Situação de Rua, previsto no Decreto № 7.053/2009, no âmbito da Assistência Social. Sendo esta uma unidade de referência da Proteção Social Especial de Média Complexidade, as ações do centro de referência devem ser articuladas intersetorialmente, contando tanto com ações da assistência social quanto de outros setores, como a previdência social, saúde, lazer, educação, cultura, trabalho, renda, dentre outros. O pleno funcionamento desse dispositivo cumpre o que é trazido pela Política Nacional para a População em Situação de Rua e deve ser associado a ações de proteção e promoção de direitos desses sujeitos, para fortalecimento de sua autonomia (BRASIL, 2011).

O Centro de referência visitado para a construção desse estudo atende a homens e mulheres maiores de idade, que chegam ao serviço por demanda espontânea ou encaminhada. O objetivo do serviço é o fortalecimento de vínculo equipe-usuários para a superação da situação de rua, por meio de acompanhamentos e encaminhamentos gerados a partir da demanda, como apoio para a obtenção de benefícios sociais e documentação; além da integração e/ou inserção o do usuário na rede de saúde. Ademais, o serviço fornece duas refeições ao dia e dispõe de espaço para que os usuários e usuárias cuidem da sua higiene pessoal.

Nesse sentido, o Projeto de Educação pelo trabalho para a Saúde - Interprofissionalidade (PETSaúde/Interprofissionalidade) de um município do Piauí, objetiva fomentar processos formativos atentos a interprofissionalidade nos cenários da universidade, serviço e comunidade, apoiando institucionalmente o município e instituições de ensino no que se refere à produção de dados e dispositivos promotores de vivências e experiências que envolvam assistência, gestão, trabalho e educação em saúde.

Este projeto de extensão é uma parceria de uma Universidade e a Secretaria Municipal de Saúde de um município do Piauí. Estão inseridos no projeto os/as alunos/as e docentes dos Cursos de Graduação em Medicina, Psicologia e Fisioterapia. Os três cursos de graduação da área da saúde da realidade em questão, apresentam suas peculiaridades formativas e potencialidades a serem melhoradas em consonância às necessidades dos territórios da saúde, integração ensino-serviço-comunidade e princípios e diretrizes do SUS.

\section{RELATO DE EXPERIÊNCIA}

Esse estudo é de caráter descritivo qualitativo, sendo possível com os recursos metodológicos da observação participante, a produção de diários de campo e das rodas de conversa. No que se refere à observação participante, compreende-se um modelo co-participativo no qual existe uma interação mais genuína entre os membros do grupo, uma vez que o pesquisador torna-se um membro efetivo e atuante do grupo ao qual está tecendo sua análise. Dessa forma, é possível obter uma série de dados comportamentais dos indivíduos e, ainda por cima, trabalhar para que a distorção resultante do fato do investigador ser o agente estranho seja reduzida ao mínimo (KLUCKHOHN FR, 2018).

Seguindo este viés, utilizamos a confecção de diários de campo, que são instrumentos frequentemente utilizados nas pesquisas de cunho qualitativo e possuem como objetivo não apenas o registro das estratégias metodológicas empregadas na condução do processo de pesquisar em saúde, mas também se constitui em um modo de compreender o objeto de estudo em suas múltiplas dimensões e inter-relações (ARAÚJO LFSD et al, 2013), ou seja, a análise foi feita tanto com base nas falas, quanto nas expressões corporais e impressões pessoais do próprio observador. 
Diante do contínuo aumento das pessoas em situação de rua, bem como dos desafios na atenção às demandas de saúde desta população, este estudo teve como principal objetivo, tecer uma análise acerca das pessoas em situação de rua em um município do Piauí, através de um relato de experiência, abordando o aspecto subjetivo apresentado nos relatos dos usuários e usuárias do Centro POP durante visitas realizadas para que, através deste novo olhar, pudessem ser planejadas e realizadas intervenções práticas que atendessem às reais necessidades desta população. Esse estudo trata-se de um relato de experiência do tipo descritivo qualitativo, obtido durante a participação dos estudantes do PETSaúde/Interprofissionalidade em rodas de conversa com usuários e usuárias de um centro de referência de um município no litoral do Estado do Piauí.

O centro de referência do município do Piauí funciona desde 2014, de segunda-feira à sexta-feira das $07 \mathrm{~h} 30 \mathrm{minh}$ às $11 \mathrm{~h} 30 \mathrm{minh}$ e das $13 \mathrm{~h} 30 \mathrm{minh}$ às $17 \mathrm{~h} 30 \mathrm{minh}$ (horário comercial). A equipe profissional do serviço conta com dois psicólogos, uma coordenadora, duas assistentes sociais, dois educadores sociais, duas auxiliares de serviços gerais, uma cozinheira, um motorista e dois guardas.

Ao entrar no centro de referência, percebemos a necessidade de conhecer melhor a população em situação para que sejam elaboradas intervenções efetivas direcionadas ao público em questão, pois só há dados em relação ao número de pessoas que frequentam o serviço, o qual representa apenas parte do total de pessoas em situação de rua da cidade. Ações eficazes na melhoria da qualidade de vida dessa população são urgentes em todo o território nacional, em especial em cidades de porte médio a grande.

Durante o período de visitas ao centro de referência, pode-se acompanhar a rotina desse dispositivo do Sistema Único de Assistência Social (SUAS) e observar, bem como interagir com alguns dos usuários e usuárias, que aceitaram participar de pequenas rodas de conversas formadas espontaneamente em cada visita. Durante as rodas de conversas foram abordadas várias questões: (1) apresentações individuais; (2) a nacionalidade; (3) informações sobre vida sexual e amorosa. Uma interação de forma descontraída e informal, gerando o mínimo de desconforto e contribuindo para maior entrosamento do grupo de estudantes com os usuários e usuárias.

A respeito das informações obtidas, percebeu-se que a maioria dos usuários e usuárias do centro de referência que participaram dos encontros é de nacionalidade brasileira, excetuando-se um, que se identificou como venezuelano. Com o passar das atividades, foi observado que poucos desses usuários reapareciam na semana seguinte, compreendemos assim, que o acompanhamento longitudinal de todos os indivíduos faz-se impossibilitado. Com referência à idade, o serviço dá suporte a usuários e usuárias que possuem a faixa etária entre 18 e 60 anos, sendo a maioria do sexo masculino. Em contrapartida, observouse também que alguns usuários não sabem ou não lembram suas datas de nascimento, evidenciando assim, a ausência de documentos de identificação de parte da população abrangida pela instituição.

Por meio de suas falas conseguimos perceber um dos grandes sofrimentos dessa população: o preconceito estabelecido contra elas, que também é observado em âmbito nacional. Por exemplo, João (nome fictício) nos expressou seu sentimento de injustiça ao relatar que durante os festejos religiosos do município, ele e outros colegas são constantemente mal interpretados e sobre si recaem inúmeras suspeitas sobre a qualidade do caráter de cada um, ou seja, sempre supõem que querem roubar os carros ou seus donos quando, na verdade, eles estão apenas atrás de uma "fonte de renda extra" ao vigiar os carros durante a noite. Ao terminar seu relato, outro membro do grupo tentou "justificar" o comportamento preconceituoso das outras pessoas: "uns fazem, nós pagamos...". Ao proferir essas palavras, percebemos que sua entonação e expressão facial nos mostraram tristeza.

\section{DISCUSSÃO}

Natalino MAC (2006) já alertava que é mais difícil realizar pesquisas com este público que com populações moradoras de domicílios particulares e/ou moradias coletivas, tais como presídios, hospitais e conventos. Ferreira CPS, Rozendo CA e Melo GB (2016) apontam que há uma realidade do grupo populacional em questão que indica para uma vivência em extrema vulnerabilidade social, em que as vidas são demarcadas por privações de direitos, rompimento de vínculos afetivos, violência, sofrimento e estigmas. 
De acordo com o Ministério de Desenvolvimento Social e Combate à Fome (2009), as pessoas em situação de rua são impedidas de acessar os serviços de saúde (18.4\%), os transportes coletivos (29.8\%), os shopping centers (31.3\%), os estabelecimentos comerciais (31.8\%) e os órgãos públicos $(21.7 \%)$.

O que se estende ao relato de João, que se sente afastado dos festejos da cidade em que reside, mas também contempla o desabafo e situação de vida de muitos outros usuários os quais tivemos contato, que necessitam do acompanhamento do centro de referência para que sejam atendidos em órgãos públicos.

Tais informações foram necessárias para compreendermos mais sobre as pessoas em situação de rua da realidade em questão, ademais, pesquisas nacionais nos mostram que as Infecções Sexualmente Transmissíveis (alta prevalência de sífilis e hepatites) estão relacionadas a fatores individuais, sociais e de saúde, atreladas a condições do contexto no qual as pessoas estão inseridas, sendo alguns grupos populacionais, a exemplo das Pessoas em Situação de Rua (PSR), considerados vulneráveis (SANTOS MVS et al., 2016; SILVA RJC et al, 2001).

Para Curvo D et al (2018) a condição rua institui marcas de estigmatização e criminalização, que se dão partir da imposição de poder instituido por saberes e práticas que permitem a inferiorização social e econômica do referido grupo, que é culpabilizado e criminalizado pelas condições as quais está esposto.

A partir desses fatos, o grupo propôs o desenvolvimento de atividades que giravam em torno da conscientização dos usuários e usuárias a respeito da importância da realização de testes rápidos para detecção de HIV, sífilis e hepatite B e C.

Ao longo da ação, detectou-se um teste reagente ao HIV. Após o ocorrido, referenciou-se o usuário para o Centro de Testagem e Aconselhamento (CTA) que é o local dentro da rede especializado em dar apoio e suporte aos pacientes com infecções sexualmente transmissíveis.

Nosso trabalho no centro de referência também esteve direcionado à aproximação dos usuários do serviço e da equipe; no sentido de que autores como Bulla LC, Mendes JMR e Prates JC (2004) já destacavam a relevância da integração dos sujeitos que estão nas ruas com as instituições que os acolhem, sendo que a função acolhedora exercida pelos trabalhadores das instituições pode ser decisiva na construção de projetos individuais e coletivos.

Assim, Costa APM $(2005$, p.14) conclui que a realização de um trabalho social que vise a segurança das pessoas e o direito à convivência e à individualidade, deve se guiar por princípios éticos de respeito à dignidade humana e à realidade de vida dessas pessoas que serão atendidas. Dessa forma, as instituições devem avaliar-se permanentemente, na busca constante de coerência.

É neste sentido que compreendemos, a partir da nossa experiência, que os seres humanos possuem necessidades, prazeres e vontades, portanto, deve-se conhecer e compreender cada uma dessas variáveis para se começar a projetar tal perfil. Identificamos o que Mattos RM e Ferreira RF (2004) destacam, em que há sim a existência de representações sociais pejorativas, em relação à população em situação de rua, que se materializam nas relações sociais.

O resultado desse estudo não é, em circunstância alguma, fixa e fechada, pelo contrário, é livre de padrões e está em constante mutação, como os indivíduos com quem se trabalhou. A perspectiva dos participantes que protagonizam esse estudo trata-se de um público que traz impregnado no seio de seu discurso, a denúncia de um preconceito, um desvalor e uma total desigualdade no tocante às formas de atendimento nos serviços públicos e recebimento de cuidados.

Entretanto, os problemas que atingem diretamente a população em situação de rua estão longe de serem apenas os relacionados à saúde/doença (SCHERVINSKI AC, et. al., 2017). Tal público declara várias vezes à sensação de invisibilidade social que sofrem nas ruas, ao serem ignorados ou tratados com desprezo, por exemplo, tal invisibilidade se revela também pela falta de documentação necessária para acessar serviços e benefícios sociais que o Estado garante (HALLAIS J e BARROS N, 2015).

Desse modo, é importante ressaltar a necessidade de garantir meios que ajudem e favoreçam na implementação de políticas públicas que sejam capazes de ampliar sua atuação e abrangência protetiva com esse público, promovendo e produzindo saúde. 


\section{REFERÊNCIAS}

1. ARAÚJO LFSD. et al. Diário de pesquisa e suas potencialidades na pesquisa qualitativa em saúde: subtítulo do artigo. Rev. Bras. Pesq. Saúde: subtítulo da revista, Vitória, Volume, Número, p. 53-61, set./2013. Disponível em: http://www.portaldepublicacoes.ufes.br/RBPS/article/viewFile/6326/4660. Acessoem: 15 set. 2019.

2. BORYSOW IC, FURTADO JP. Acesso e intersetorialidade: o acompanhamento de pessoas em situação de rua com transtorno mental grave. Physis: Revista de Saúde Coletiva, v. 23, p. 33-50, 2013.

3. BRASIL; BRASIL. Decreto n 7.053, de 23 de Dezembro de 2009. Institui a Política Nacional para a População em Situação de Rua e seu Comitê Intersetorial de Acompanhamento e Monitoramento, e dá outras providências. Diário Oficial [da] União, 2009.

4. BRASIL. Departamento de Proteção Social Especial. Orientações Técnicas: Centro de Referência Especializado para População em Situação de Rua - Centro Pop. Gráfica e Editora Brasil LTDA, Brasília, 3 ed. 2011.

5. BULLA LC, MENDES JMR, PRATES JC. (Orgs.). As múltiplas formas de exclusão social. Porto Alegre: Federação Internacional de Universidades Católicas: EDIPUCRS, 2004.

6. COSTA APM. População em situação de rua: contextualização e caracterização. Textos \& Contextos (Porto Alegre), v. 4, n. 1, p. 1-15, 2005.

7. CURVO D, MATOS A, SOUSA W, PAZ, A. Integralidade e clínica ampliada na promoção do direito à saúde das pessoas em situação de rua. Cadernos Brasileiros de Saúde Mental/Brazilian Journal of Mental Health, 2018.

8. FERREIRA CPS, ROZENDO CA, MELO GB. Consultório na Rua em uma capital do Nordeste brasileiro: o olhar de pessoas em situação de vulnerabilidade social. Cadernos de Saúde Pública, v. 32, p. e00070515, 2016.

9. FERRO MCT. Política Nacional para a População em Situação de Rua: o protagonismo dos invisibilizados. Revista Direitos Humanos, v. 8, p. 35-39, 2012.

10. HALLAIS J, BARROS N. Consultório de rua: visibilidades, invisibilidade e hipervisibilidade. Cadernos de Saúde Pública, Rio de Janeiro, v. 31, n. 7, jul. 2015.

11. IBGE, IBGE. Cidades. Avaliable from: http://www. ibge. gov. br/cidadesat/topwindow. htm, v. 1, 2010.

12. KLUCKHOHN FR. O Método de Observação Participante no Estudo de Pequenas Comunidades. Sociabilidades Urbanas. Revista de Antropologia e Sociologia, v2, n5, p. 29-38, julho de 2018. ISSN 2526-4702.

13. MATTOS RM, FERREIRA RF. Quem vocês pensam que (elas) são?-Representações sobre as pessoas em situação de rua. Psicologia \& Sociedade, v. 16, n. 2, p. 47-58, 2004.

14. Ministério do Desenvolvimento Social e Combate a Fome. Rua aprendendo a contar: pesquisa nacional sobre a população em situação de Rua. Brasília: MDS. 2009.

15. NATALINO MAC. Estimativa da população em situação de rua no Brasil. Texto para Discussão, 2016.

16. PIMENTA MM. Pessoas em situação de rua em Porto Alegre: Processos de estigmatização e invisibilidade social. Civitas-Revista de Ciências Sociais, v. 19, n. 1, p. 82-104, 2019.

17. SANTOS MVS et al. Sexo Obrigado: cuidados de pessoas em situação de rua para infecções sexualmente transmissíveis. V Seminário Internacional Enlaçando Sexualidades, 2016.

18. SCHERVINSKI AC et al. Atenção à saúde da população em situação de rua. Extensio: Revista Eletrônica de Extensão, Florianópolis, v. 14, n. 26, p. 55-64, nov. 2017.

19. Silva RJC et al. Syphilis in a homeless population in Sao Paulo, Brazil. In: International Congress of Sexually Transmitted Infections, ISSTDR/IUSTI; 2001 jun 24-27 Berlin (AI). Int J of STD \& AIDS 2001; 12: 136-7.

20. VIEIRA MC, BEZERRA EMR, ROSA CMM. (Orgs.). População de rua: quem é? Como vive? Como é vista? São Paulo: Hucitec, 1994. 